Methods and Materials: This retrospective study included children who underwent EDAS over 7-year period (May 2012-May 2019). All the necessary data were collected from computerized case records, inpatient hospital records and anesthesia records.

Results: A total of 45 children who underwent 52 procedures of EDAS for Moyamoya disease were identified with a median age of 8 years (IQR: 4.5-11.5), male preponderance $(M: F=25: 20)$, median body weight of $30 \mathrm{~kg}$ (IQR: $18.25-42$ ), and mean BMI of $19.2 \pm 5.6 \mathrm{~kg} / \mathrm{m}^{2}$. All children (100\%) had previous stroke at presentation with 2 years (IQR: 1-4) median duration of symptoms at presentation. They received GA with controlled ventilation using air, oxygen, sevoflurane, or isoflurane and regional scalp block using $0.2 \%$ bupivacaine $/ 0.2 \%$ ropivacaine for analgesia at the beginning and end of surgery. Hemodynamics were maintained within the baseline. Eight (15.4\%) children developed neurological deficits following surgery, among which five had permanent deficits and three had transient deficits which improved with hemodynamic management and hydration. The baseline parameters, possible risk factors, and outcome variables between the patients who developed postoperative deficits and those who did not develop postoperative deficits were compared.

Conclusions: Preoperative hydration and postoperative analgesia with intravenous fentanyl and scalp block reduced the incidence of postoperative stroke.

\section{A004 Comparison of Hemodynamic Profile of Ketamine and Propofol (Ketofol) with Propofol in Traumatic Brain Injury Patients Undergoing General Anesthesia Neha Maheshwari, ${ }^{1}$ Nidhi Panda, ${ }^{1}$ Nidhi Bhatia, \\ Ankur Luthra' \\ 'Department of Anaesthesia and Intensive care, Post graduate Institute of Medical Education and Research (PGIMER), \\ Chandigarh, India}

Background: Comparison of MAP, brain relaxation, plasma level of glial fibrillary acidic protein (GFAP) in intraoperative and extended Glasgow outcome scale (GOSE) at 3 months of postoperative period in patients receiving "ketofol" (ketamine and propofol $=1: 5$ ) or propofol for induction and maintenance of anesthesia in TBI patients undergoing surgery.

Materials and Methods: A prospective randomized controlled trial was done on 50 TBI patients undergoing surgery after approval of Institute Ethics Committee and written informed consent from patients' kin. Patients in group $\mathrm{P}(n=25)$ received propofol, while patients in group KP $(n=25)$ received combination of ketamine and propofol (1:5) for induction and maintenance of anesthesia. Invasive MAP was recorded continuously. Rescue measure to maintain MAP were recorded. Brain relaxation, ONSD, plasma biomarker GFAP, and GOSE (at 3 months) were observed.

Results: Throughout the drug infusion period MAP was better maintained and hemodynamic fluctuations were less in patients receiving ketofol compared with those receiving propofol $(p<0.05)$. There was decreased requirement of phenylephrine in ketofol group $(p=0.02)$. There is no statistically significant difference in quality of brain relaxation, ONSD, GFAP values, number of ventilator days, and GOSE score at 3 months in either group.

Conclusions: Ketofol (ketamine and propofol in the ratio 1:5) is preferred over propofol as induction and maintenance anesthetic agent in patients undergoing surgery for TBI as it provides more hemodynamic stability compared with propofol alone with no significant adverse effects.

\section{A005 Correlation of Neutrophil-to-Lymphocyte Ratio and Platelet-to-Lymphocyte Ratio with Neurological Outcome in Moderate and Severe Traumatic Brain Injury Patients: Do they Improve upon the TBI Impact and Crash Diagnostic Models? \\ Kanchan Bilgi, ${ }^{1}$ Gopalakrishna K.N., ${ }^{1}$ \\ Dhritiman Chakrabarti, ${ }^{1}$ G.S. Umamaheswara Rao ${ }^{1}$ \\ 'Department of Neuroanesthesiology and Neurocritical \\ Care, National Institute of Mental Health and Neuro Sciences \\ (NIMHANS), Bengaluru, Karnataka, India}

Background: We proposed to study (1) the correlation of neutrophil-lymphocyte ratio (NLR) and platelet-lymphocyte ratio (PLR) with the neurologic outcome in moderate-to-severe head injury patients and (2) whether they improve the performance of the validated prognostic models available at present.

Materials and Methods: This prospective cohort study, conducted in a tertiary care neurosurgical center after ethical clearance, included patients, aged 18 to 60 years with moderate and severe isolated head injuries. Laboratory and clinical parameters were noted at admission and the extended Glasgow outcome score (GOSE) of the patients was assessed after 6 months. IMPACT and CRASH models were used to predict the outcome. Logistic regression, Pearson's correlation, Spearman's rank and Chi-square tests were used for analysis.

Results: Among the 96 patients included for analysis, the incidence of poor outcome was $59.38 \%$ and the mortality was $43.8 \%$. The ability of NLR (AUROC $=0.58)$, PLR $(A U R O C=0.58)$, and both combined (AUROC $=0.53)$ in predicting mortality, as well as neurologic outcome (AUROC: $\mathrm{NLR}=0.47$; PLR $=0.53$; combined $=0.56$ ) was insignificant. The agreement between the independently predicted neurologic outcome and mortality by NLR and PLR with IMPACT (NLR: $p=0.74$; PLR: $p=0.39$ ) and CRASH (NLR: $p=0.45$; PLR: $p=0.79$ ) models was poor.

Conclusions: NLR and PLR did not predict the neurologic outcome in moderate to severe head injury patients. Addition of these parameters to the standard TBI-IMPACT and CRASH prognostic models did not improve the predictive value of the models. In our study, the earlier validated prognostic models seem to have better predictive value than the NLR and PLR. 